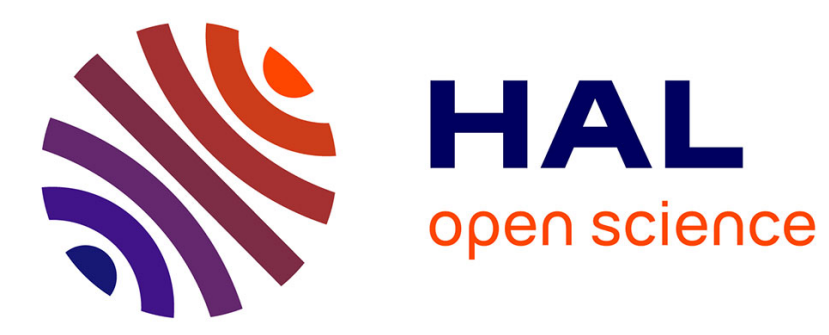

\title{
Modeling and control of a piezoelectric microactuator with proprioceptive sensing capabilities.
}

Roba El Khoury Moussa, Mathieu Grossard, Mehdi Boukallel, Arnaud

Hubert, Nicolas Chaillet

\section{- To cite this version:}

Roba El Khoury Moussa, Mathieu Grossard, Mehdi Boukallel, Arnaud Hubert, Nicolas Chaillet. Modeling and control of a piezoelectric microactuator with proprioceptive sensing capabilities.. Mechatronics, 2014, 24, pp.590-604. 10.1016/j.mechatronics.2013.12.002 . hal-01303500

\section{HAL Id: hal-01303500 \\ https://hal.science/hal-01303500}

Submitted on 18 Apr 2016

HAL is a multi-disciplinary open access archive for the deposit and dissemination of scientific research documents, whether they are published or not. The documents may come from teaching and research institutions in France or abroad, or from public or private research centers.
L'archive ouverte pluridisciplinaire HAL, est destinée au dépôt et à la diffusion de documents scientifiques de niveau recherche, publiés ou non, émanant des établissements d'enseignement et de recherche français ou étrangers, des laboratoires publics ou privés. 


\title{
Modeling and Control of a Piezoelectric Microactuator with Proprioceptive Sensing Capabilities
}

\author{
Roba El Khoury Moussa ${ }^{1}$, Mathieu Grossard ${ }^{1}$, Mehdi Boukallel ${ }^{2}$, Arnaud Hubert ${ }^{3}$, and Nicolas Chaillet ${ }^{3}$ \\ Corresponding author: e-mail: mathieu.grossard@cea.fr, tel: +33(0)169080787, fax: +33(0)169080701 \\ ${ }_{1}^{1}$ Interactive Robotics Laboratory, LIST, CEA, Gif-sur-Yvette, F-91190, France \\ 2 Sensorial and Ambient Interfaces Laboratory, LIST, CEA, Gif-sur-Yvette, F-91190, France \\ ${ }^{3}$ Automatic Control and Micro-Mechatronic Systems Department, FEMTO-ST Institute, Université de Franche-Comté, ENSMM-UTBM-CNRS, \\ Besançon, France
}

\begin{abstract}
In this paper, modeling and control strategies for a new observability-optimized piezoelectric microactuator are presented. The targeted applications concern mainly the design of microgripper for micromanipulation tasks. The device has been designed using a topological optimization method, which takes into account the optimal full integration of piezoelectric actuating and sensing elements within the device. It is achieved in link with modal controllability and observability considerations. The vibrational modes that govern the tip deflection of the monolithic compliant structure are proved to be fully observable by the integrated sensing area of the device. The proposed control strategy permits to simply reconstruct the deflection using electric charges measurement and modal state observer. Finally, the vibrations naturally induced by the flexible structure are successfully damped using robust and low order controller.
\end{abstract}

Keywords: Flexible structure, piezoelectric microactuator, vibration control, topological optimization.

\section{Introduction}

Micromanipulation tasks use mostly microgripper devices based on compliant mechanisms. These ones are monolithic flexible structures that transmit a motion under elastic strain. Compliant mechanisms present many advantages at the small scale: simplified manufacturing, reduced assembly costs, reduced noise, no wear, no backlash, high precision, and ability to accommodate unconventional actuation schemes. One interesting type of smart material used in microrobotic applications is the piezoceramic PZT. Piezoelectric actuation has become widespread in micromanipulation systems where high positioning accuracy is needed [1]. Beyond their appealing properties in the sense of micromechatronic design, an advantage of using piezoelectric material is its reversible electromechanical effect. This explains its high potential use in the microrobotic field as actuators and/or sensors $[2,3]$. The design of smart microrobotic structures based on piezoelectric material, or more generally on active materials, is a non-intuitive task because of their multifunctional requirements (Fig. 1). This task poses the problem of the physical integration of actuators and sensors in the compliant mechanism (size, topology, ...), as well as their optimal placements in the full structure [4]. Indeed, these locations influence directly the performances that can be reached by the device, even if feedback or feedforward control strategies are used [5].

Many scientific works have already been done on the problem of optimally distributing piezoelectric actuating and sensing elements within flexible structures. They deal mainly with active vibration control or minimization of undesired spillover effects $[6,7,8]$. In particular, topology optimization solves the problem of distributing reference material in a design domain subjected to loading and support conditions. Although various topology optimization methods have been applied to the design of piezoelectric devices $[9,10]$, most of them consider the optimal distribution of passive materials while predefining the locations of piezoelectric materials. Other works are focused on the optimal design of coupling structures for piezoelectric actuator, considering maximum energy efficiency [11]. But finally, few studies deal with the optimal distribution of piezoelectric materials for the design of monolithic piezoelectric actuator using topology optimization formulation [12,13] and even less for piezoelectric sensor design [14]. To our knowledge, no topological method have been yet used for the topological optimization of monolithic piezoelectric 


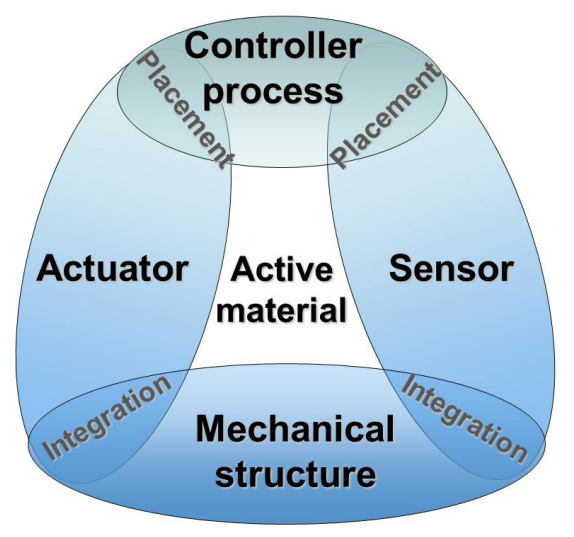

Figure 1: Smart structure design.

devices with a fully integration of both actuation and sensor components. In addition, very few related works deal with topological optimization method for actuator or sensor design including frequency response analysis [12, 15]. In these related works, the generally used objective functions are based on the maximization of either geometrical advantage (stroke amplification), or mechanical advantage (force amplification), according to predefined harmonic loadings. Optimization of these active compliant micromechanisms from the first design step will strongly improve their performances if we take into account relevant criteria from the control field.

In previous works, a new compliant PZT monolithic microactuator was designed in simulation by authors [16]. Its flexible structure has been simulated and optimized using a multidisciplinary topology optimization algorithm. The software design method is based on a building block method and a genetic algorithm that optimize the passive, active and sensitive areas of the flexible structures. In this paper, the device has been prototyped and its observer-based controller is presented. In addition to purely mechanical criteria, innovative observability-based considerations are taken into account in our design. This ensures the effectiveness of the control strategies presented in the following. It relies on observability-based criteria which exploit the modal gramians properties of flexible structures [17]. A special attention has been paid on the optimal physical integration of the actuating and proprioceptive sensing areas within the structure regarding dynamic performances. Indeed, the tip displacement $\delta$ to be controlled have to be optimally reconstructed from induced charges $q$ measurements in the sensitive structure. Finally, the strategy we proposed in this paper avoids the use of exteroceptive laser sensor normally required for accurate closed loop control of the deflection.

This paper is organized as follows. In section II, we briefly review the underlying idea of the observability-based optimization strategy. It led to the specific design of the microactuator subsequently prototyped and presented in section III. In section IV, the experimental vibrational dynamics governing the tip deflection and the electric charges induced in the prototype are validated according to the optimization specifications. In section $\mathrm{V}$, nonlinear phenomena are considered from the perspective of control design. We construct the complete electromechanical model of the tip deflection $\delta$ and the electric charges $q$ as a function of the input control voltage $u$. The hysteresis phenomenon in deflection is compensated using a Prandl-Ishlinskii (PI) technique and a damping cascaded controller is implemented. Finally, a modal state observer is adopted to reconstruct the dynamics of the tip deflection from the proprioceptive sensor. The control of the reconstructed tip deflection is experimentally performed.

\section{Strategy for observability-based design of flexible structures}

In this section, observability issues are considered together with the multiobjective optimization procedure.

\subsection{Observability issues}

The design of active structures with both integrated actuation and sensor capabilities is a non-intuitive task and the designer is faced with two main issues: 
1. A reduced model of the structure has to be synthesized, which must include the few dominant low-frequency modes, without rejecting the residual modes that can destabilize the system (i.e. high roll-off after the dominant modes). Resonance peaks amplitudes must be maximized in the frequencies bandwidth to increase authority control on the dominant modes of the deflection transfer function $\delta / u$. On the contrary, the amplitudes of resonance peaks after cut-off frequency must be minimized to increase gain margin and to limit modes destabilization in this area (spillover phenomenon).

2. If all dominant modes are not well observed by the sensing area of the flexible structure, the reconstruction of the deflection $\hat{\delta}$ will not be guaranteed in an optimal way by the observer.

Hence, the authors propose in [16][18] a new numerical criterion, referred as $J^{k, t h v}$ in the following, to meet these two issues: the first problem disappears when the pseudo-optimal structure is forced to have $k$ first dominant modes in the $\delta / u$ control transfer function $G_{c o}(s)$ with $s$ the complex variable of the Laplace domain (see Fig. 2). The second problem is overcame if we guarantee a high-level of observability of these dominant modes by means of the electric charges in the observation $q / u$ transfer function $G_{o b}(s)$. This new criterion is applied to the optimal design of a new microactuator device, and this is the core of this paper.

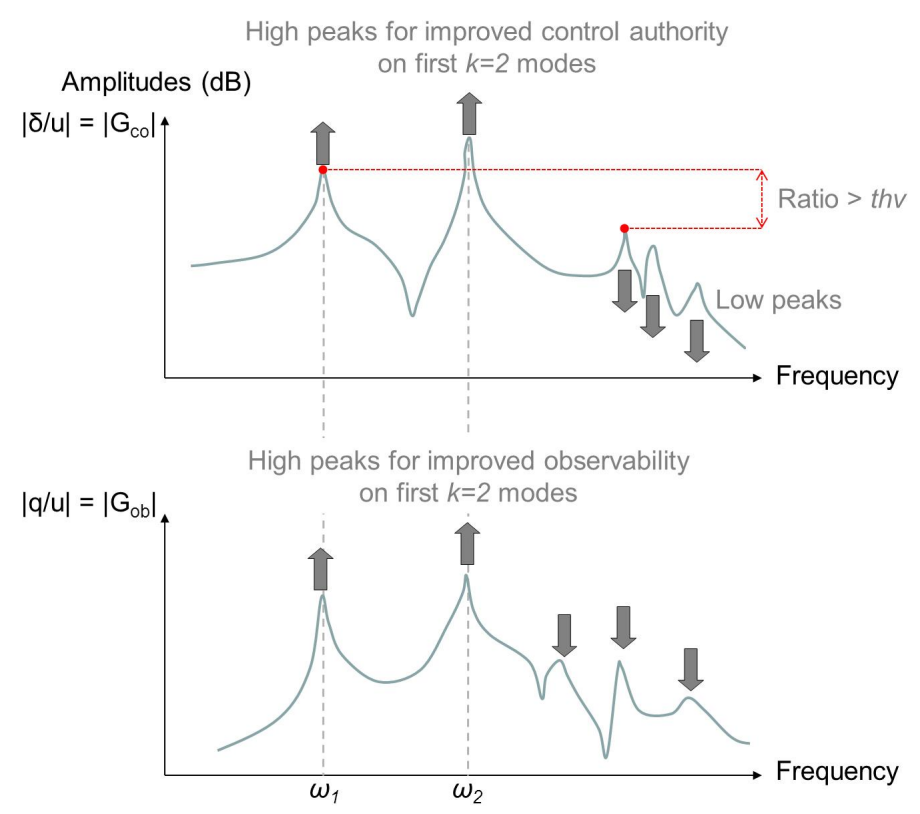

Figure 2: Example of desired forms for the control $G_{c o}$ and observation $G_{o b}$ transfer functions.

With thv a threshold value specified by the designer, the optimization procedure ensures that the $k$ first modes dominate by at least thv times the residual modes (as seen in Fig. 2). In other words, when satisfying this criterion, we privilege the modes where good observability of $\delta / q$ operator coincides with its dominant modes.

\subsection{Optimization methodology for integrated piezoelectric device}

The above criteria were used with a multidisciplinary topology optimization procedure. Reader can find detailed descriptions of the building block optimization tool in [16].

This optimization tool is based on a multiobjective genetic algorithm approach, which searches the optimal distribution of available building blocks. The resulting structure is thus an assembly of elementary passive, active or sensitive compliant blocks chosen from a library. Some blocks can act as pure mechanical stiffness, whereas others integrate actuation or sensing functions. The optimization problem not only takes into account the topological specifications, but also an optimal set of boundary conditions (fixed frame location, contacts, end-effectors, etc), dimensions and materials, depending on some optimization criteria selected by the designer.

The library of building blocks is composed of 36 building block elements, made of beams assembly. They are sufficient to build a high variety of topologies. Moreover, the block feasibility related to fabrication process constraints 
can also be taken into account during the problem specification. The blocks present some various topologies. Their advantage is that they can furnish multiple coupled DoFs, thus generating more complex movements with only one building block than a method which would have consisted in optimizing directly the location of the elementary beams of the truss-like structure. Thus, the function of the building blocks method is to save computational time during the optimization process by reducing complexity of the combinatorial of the problem. It should also be noted that calculation of the different matrices of each valued block is done one time only at the beginning of the optimal design problem (before running the genetic algorithm), which saves running time.

From the static point of view and for an applied external voltage $u$, the whole topology of the flexible structure is designed to maximize both the steady-state tip deflection $\delta$ and the electric charges $q$ on the sensing parts. In addition to these pure mechanical criteria, we consider the $J^{k, t h v}$ criterion with $k=2$ and $t h v=3$ for the pseudooptimal design of the microactuator structure presented in this paper. This criterion enables to shape the open-loop frequency responses of both the controlled deflection $\delta / u$ transfer operator and observed electric charges $q / u$ transfer operator as described previously. From the topological point of view, this results in an appropriate placement of the actuating and sensing areas. Used together in a multiobjective optimization procedure, these three criteria can fully and pseudo-optimally integrate the actuation and sensor functions into the structure.

\section{Presentation of the observability-optimized piezoelectric prototype}

The pseudo-optimal prototyped microactuator is presented in the following. The proposed control strategy is also introduced.

\subsection{Overview of the microactuator prototype}

The monolithic micromechatronic prototype results from an assembly of PZT beams (Fig. 3). Either passive, active or sensitive beams inside a block-like mesh constitutes the compliant structure. It is assumed to undergo structural planar strain, mainly due to in-plan flexion. The structure topology has a maximal size of $15 \mathrm{~mm} \times 18 \mathrm{~mm}$, and a constant thickness of $200 \mu \mathrm{m}$.

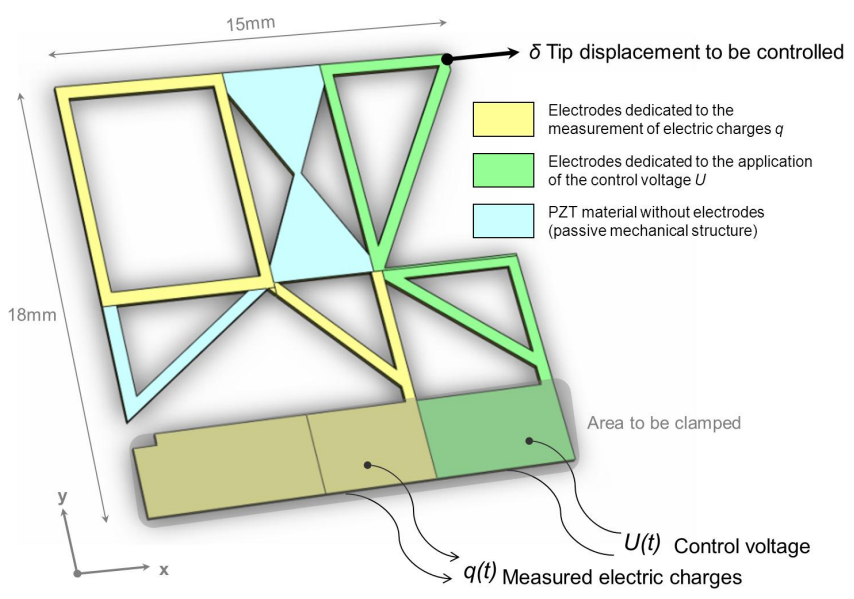

Figure 3: 3D CAD of the left-finger PZT microactuator with integrated sensing capabilities.

Our approach consists in integrating a voltage driven actuator and a charge measuring device for sensing. Even if other arrangements are also possible (e.g. charge driven actuation [19]), ours has been widely used in the control of flexible structures and seems to be the most straightforward way to take into account actuation and sensing in our optimized Finite Element modeling. Indeed, such a configuration enables the structural mechanical dynamics of each structure to be easily computed on-line, avoiding modification of mechanical poles locations due to specific charge driven considerations. Regarding the actuated beams, longitudinal strain is generated under the transverse electric field along the thickness, thus exploiting the transverse effect of piezoelectricity. Regarding the sensitive beams, they exploit the piezoelectric effect to generate electric charges when submitted to the strain field of the actuated flexible 
structure. An electronic charge-voltage converter is then used to extract the electric charges from the sensing areas. If ideal operating mode of this electronic converter is supposed, upper and lower electrodes of the sensing piezoelectric beams are assumed short-circuited. As a consequence, the sensing piezoelectric beams are only linked to the local strain of the microactuator.

The monolithic structure can be considered as the actuator of a left-arm gripper and is made of a single piezoelectric material PIC151 from PI Piezo Ceramic Technology. This device includes either passive, active or sensitive blocks inside a $2 \times 3$ mesh [16]. In the optimization procedure, the number of active (resp. sensitive) blocks in the structure could vary between 1 and 4 . They are optimally chosen in the compliant building block library. When external voltages are applied to the blocks electrodes, the output node (tip of the structure) has to move along the $x$-axis. The size ratio of the blocks can vary between 1 and 2 . The boundary conditions of possibly fixed nodes are exclusively devoted to the parts that are reported in the bottom line of the structure. When the genetic algorithm does not find any new pseudo-optimum during 200 subsequent generations (stagnation criteria), the best compromises are kept and can be found on Pareto fronts. The population size is fixed to 100 solutions, with a mutant proportion in the population of $60 \%$ and probability of genes mutation of $40 \%$. Considering mutation process within the iterative algorithm enables to avoid locally pseudo-optimal solutions.

The whole prototyped device is laser-cut from a monolithic PIC151 piezoelectric plate with electrodes on upper and lower faces (Fig. 4).

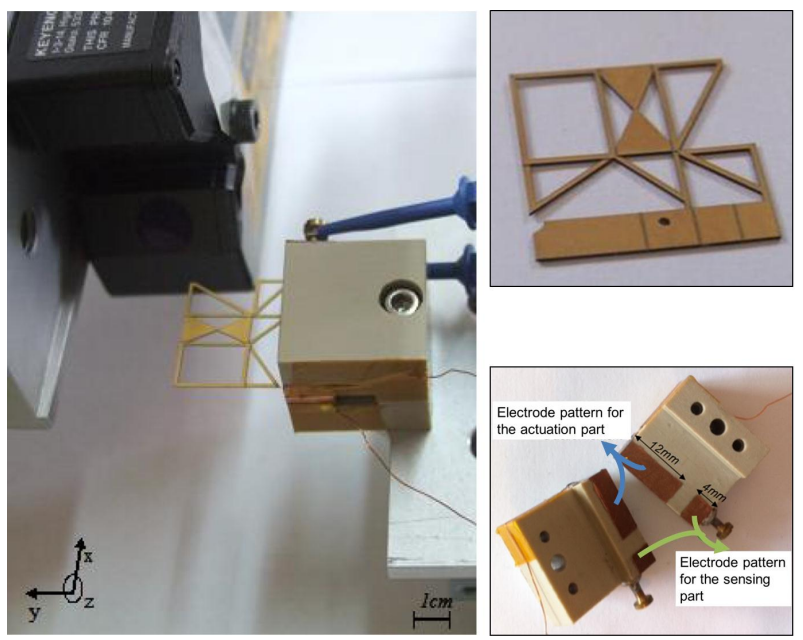

Figure 4: Machined piezoelectric device, clamping system and electrode patterns for the actuating and sensing areas.

Actuation and sensor functions are wired separately by two independent optimized electrodes paths. When external voltages are applied to the actuation electrodes $( \pm 100 \mathrm{~V})$, the whole structure deforms to produce a motion of the output node of the structure (finger tip of the microgripper) $\delta$ along the $x$-axis (max $\pm 7 \mu m)$, while the sensitive electrodes generate electric charges proportional to the strain field within the structure $\left(\max 1.9 \times 10^{-9} \mathrm{C}\right)$.

From a computational point of view, optimization criteria take advantage of the dynamic finite elements formulation of the flexible structure. This is rewritten as a finite-dimensional linear system :

$$
\left\{\begin{array}{l}
\mathbf{M} \ddot{\eta}+\mathbf{K} \eta=\mathbf{E} u \\
y_{c o}=\delta=\mathbf{F} \eta \\
y_{o b}=q=\mathbf{L} \eta
\end{array}\right.
$$

The foregoing second-order differential matrix equations represent the undamped dynamic behavior of the system, where $\mathbf{M}$ and $\mathbf{K}$ are the structure's mass and stiffness matrices. These matrices are obtained from the finite element formulation by the assembly of the matrices of all the beams constituting the structure. Let us consider the integer $p$ as the number of DoFs of the structure, $\eta$ is then the $p \times 1$ nodal displacement vector and $u$ is the input vector 
(control applied voltage on the device). The input matrix $\mathbf{E}$ reflects the location of the actuated DoFs, while $y_{c o}$ is the controlled output representing the tip displacement $\delta$ of our microgripper. The output displacement matrix $\mathbf{F}$ reflects therefore the node position of the output $y_{c o}$ among all the $p$ nodes of the truss-like structure. The third equation in (1) computes the electric charges of all the sensing beams, while the output matrix $\mathbf{L}$ points out the placement of the piezoelectric sensing beams within the structure. In this model, it is important to emphasize that the controlled output variable $y_{c o}$ is not directly observed, as compared with related works where $y_{o b}=y_{c o}$, and where the controlled tip of the piezocantilever have to be directly measured with an exteroceptive position sensor.

\subsection{Observation strategy}

To highlight the advantage of the proposed observation strategy, the physical coordinate base expansion (1) is firstly transformed into the modal base to emphasize the flexible modes contribution. The harmonic solutions of the first equation in (1) give the eigenvectors matrix $\boldsymbol{\Psi}$ and natural frequencies $\omega_{n_{i}}$ of the system :

$$
\left\{\begin{array}{l}
\ddot{\mathbf{z}}+\operatorname{diag}\left(2 \xi_{\mathrm{i}} \omega_{n_{i}}\right) \dot{\mathbf{z}}+\operatorname{diag}\left(\omega_{n_{i}}^{2}\right) \mathbf{z}=\Psi^{t} \mathbf{E} u \\
y_{c o}=\delta=\mathbf{F \Psi} \mathbf{z} \\
y_{o b}=q=\mathbf{L} \Psi \mathbf{z}
\end{array}\right.
$$

$\mathbf{z}$ is the $p \times 1$ modal displacement vector, and $\xi_{i}$ is the $i^{\text {th }}$ modal damping ratio introduced using Basil's hypothesis. One interesting $2 p \times 1$ state vector $\mathbf{x}$ typically used for flexible structures uses modal velocities and frequency-weighted modal displacements [20]:

$$
\mathbf{x}=\left(\begin{array}{lllll}
\dot{z}_{1} & \omega_{n_{1}} z_{1} & \ldots & \dot{z}_{p} & \omega_{n_{p}} z_{p}
\end{array}\right)^{t}
$$

Since controlled and observed output vectors are not the same, the modal state-space representation can be written as follows:

$$
\left\{\begin{array}{l}
\dot{\mathbf{x}}=\mathbf{A x}+\mathbf{B} u \\
y_{c o}=\mathbf{C}_{\mathbf{c o}} \mathbf{x} \\
y_{o b}=\mathbf{C}_{\mathbf{o b}} \mathbf{x} .
\end{array}\right.
$$

It gives two matrices triplets $\left(\mathbf{A}, \mathbf{B}, \mathbf{C}_{\mathbf{c o}}\right)$ and $\left(\mathbf{A}, \mathbf{B}, \mathbf{C}_{\mathbf{o b}}\right)$, one for the control and one for the observation state space models. The matrices take the forms $\mathbf{A}=\operatorname{diag}\left(\mathbf{A}_{1}, \ldots \mathbf{A}_{\mathbf{p}}\right), \mathbf{B}=\left(\mathbf{B}_{1}^{t}, \ldots \mathbf{B}_{\mathbf{p}}^{t}\right)^{t}, \mathbf{C}_{\mathbf{c o}}=\left(\mathbf{C}_{\mathbf{c o}_{1}}, \ldots \mathbf{C}_{\mathbf{c o}}\right)$, and $\mathbf{C}_{\mathbf{o b}}=$ $\left(\mathbf{C}_{\mathbf{o b}_{\mathbf{1}}}, \ldots \mathbf{C}_{\mathbf{o b}}\right)$, with, for $i=1, \ldots, p$,

$$
\begin{gathered}
\mathbf{A}_{\mathbf{i}}=\left[\begin{array}{cc}
-2 \xi_{i} \omega_{n_{i}} & -\omega_{n_{i}} \\
\omega_{n_{i}} & 0
\end{array}\right] \\
\mathbf{B}_{\mathbf{i}}=\left[\begin{array}{c}
\mathbf{b}_{\mathbf{i}} \\
\mathbf{0}
\end{array}\right] \\
\mathbf{C}_{\mathbf{c o}_{\mathbf{i}}}=\left[\begin{array}{ll}
\mathbf{0} & \frac{\mathbf{c}_{\mathbf{c o}_{\mathbf{i}}}}{\omega_{n_{i}}}
\end{array}\right] \\
\mathbf{C}_{\mathbf{o b}_{\mathbf{i}}}=\left[\begin{array}{ll}
\mathbf{0} & \frac{\mathbf{c}_{\mathbf{o b}_{\mathbf{i}}}}{\omega_{n_{i}}}
\end{array}\right]
\end{gathered}
$$

where $\mathbf{b}_{\mathbf{i}}, \mathbf{c}_{\mathbf{c o}}$, and $\mathbf{c}_{\mathbf{o} \mathbf{b}_{\mathbf{i}}}$ are the $i^{\text {th }}$ components of $\Psi^{t} \mathbf{E}, \mathbf{F} \Psi$ and $\mathbf{L} \boldsymbol{\Psi}$ respectively. The matrix $\mathbf{A}$ depends on structural parameters (mass, stiffness and damping ratio), whereas matrix $\mathbf{B}$ depends only on the location of actuated DOF, matrix $\mathbf{C}_{\mathbf{c o}}$ on the location of desired displacement output, and matrix $\mathbf{C}_{\mathbf{o b}}$ on the location of integrated piezoelectric sensors. With these notations, the targeted open-loop $\delta / u$ and $q / u$ transfer functions that were presented previously on Fig. 2 are expressed as follows:

$$
\begin{aligned}
G_{c o}(s) & =\mathbf{C}_{\mathbf{c o}}(s \mathbf{I}-\mathbf{A})^{-1} \mathbf{B} \\
G_{o b}(s) & =\mathbf{C}_{\mathbf{o b}}(s \mathbf{I}-\mathbf{A})^{-1} \mathbf{B}
\end{aligned}
$$

Based on the dominant modes considerations for the tip deflection, state observer can be tuned easily to estimate the tip deflection dynamics (Fig. 5). 


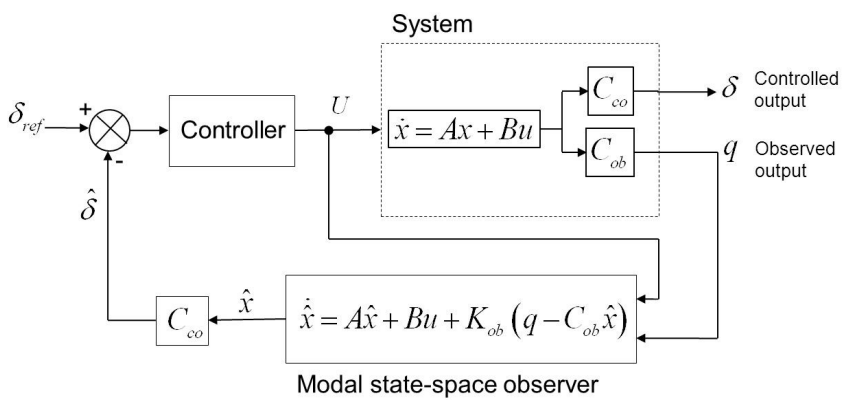

Figure 5: Diagram for the observer-based control of the compliant microactuator with integrated piezoelectric actuator and sensor $\left(K_{o b}\right.$ denotes the state observer matrix).

\section{Identification of the vibrational dynamics and interests of observation criterion for proprioceptive measure- ment}

In this section, the model is identified experimentally and compared with the linear vibrational dynamics of the transfer functions $G_{c o}(s)$ and $G_{o b}(s)$. An analysis is conducted regarding the $J^{k, t h v}$ criterion.

\subsection{Experimental setup}

The microsystem prototype is clamped, and fixed on a $x-y-z$ manual micropositioning linear stage. As piezoelectric actuators require high voltage to provide micrometric deflection, the device is supplied by a linear power amplifier, with an $\times 50$ amplification ratio (see Fig. 6).

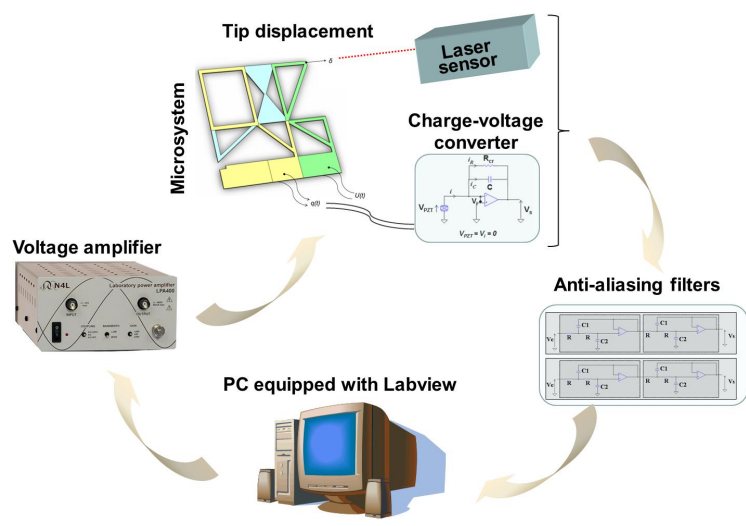

Figure 6: Schematics of the experimental setup.

The device is computer-controlled with a PC embedding a Matlab-Simulink software and a NI Labview PXI board. Output displacement of the tip is measured along the $x$-axis using a $0.01 \mu \mathrm{m}$-resolution Keyence laser sensor (see Fig. 4). In addition, an electronic charge-voltage converter is used to measure the electric charges from the sensing areas. The analog outputs of laser sensor and charge amplifiers are connected to a $4^{\text {th }}$-order low-pass antialiasing filter. Double Sallen-Key electronic circuits are tuned to provide more than $75 \mathrm{~dB}$ attenuation at $f_{e} / 2=10 \mathrm{kHz}$ Shannon frequency ( $f_{e}=20 \mathrm{kHz}$ is the sampling frequency). These devices filter high frequency components to avoid aliasing problems or unmodeled high-frequency noise dynamics.

The measurement of the tip deflection will be firstly performed using exteroceptive displacement sensor (laser sensor). It will enable us to characterize the electromechanical model of the tip deflection as a function of the input control voltage. Then, taking advantage of the proposed observability-based criterion, we will adopt a control scheme with a modal state observer based on proprioceptive sensing property of our pseudo-optimally-design device. The observer-based control strategy will exploit the electric charge measurement to efficiently reconstruct the tip deflection. Exteroceptive laser sensor will no longer be required for controlling our pseudo-optimally-design device. 


\subsection{Characterization of the deflection transfer operator}

The piezoelectric model of the device is written, considering that the applied voltage on the upper and lower electrodes of the microactuator is the input of the system. The piezoelectric behavior is considered firstly as linear although, in a general way, the deflection is non-linearly linked to external applied voltage $u$. Non-linear effect are mainly due to hysteresis effects and creep phenomena [21]. In this section, specifically dedicated to the vibrational dynamics, the nonlinearities are not taken into account, because some specific experimental precautions were considered for this purpose (nonlinear phenomena will be studied in the following sections):

- To isolate the response due to the induced vibrations from the creep phenomenon, relatively high-frequency inputs are used. The next section will prove that the transient part of piezoelectric microactuators response is shorter than $300 \mathrm{~ms}$ whereas the creep settling time is longer than $180 \mathrm{~s}$.

- Hysteresis effects could be disregarded if the voltage amplitudes are kept small [21].

A low-amplitude sine voltage input $u$ of increasing frequency is applied to the piezoactuator, where the vibrational dynamics are recorded experimentally. The tip deflection is also recorded during this experiment using the laser sensor. Bode diagram and the corresponding transfer functions were directly identified in frequency domain using a spectrum analyzer device (HP3562A). As expected by our pseudo-optimal design, the first two resonant modes are dominant among the higher frequencies residual modes (Fig. 7). The first point of interest of the criterion $J^{2,3}$ for the design is then validated. A reduced model of $G_{c o}(s)$, considering only these two dominant modes, can therefore be identified confidently. We consider a truncated second-order modal transfer expansion of $G_{c o}(s)$ of the form:

$$
\sum_{i=1}^{2} \frac{k_{c_{i}}}{1+\frac{2 \xi_{i}}{\omega_{n i}} s+\frac{1}{\omega_{n i}^{2}} s^{2}}=\frac{N_{c o}(s)}{D(s)}
$$

Damping ratios $\xi_{1}=2.0 \%$ and $\xi_{2}=2.5 \%$ are estimated from the frequency response using the measured quality factor at $-3 \mathrm{~dB}: Q_{i}^{-3 d B} \approx \frac{1}{2 \xi_{i}}$. Natural pulsations $\omega_{n_{1}}$ and $\omega_{n_{2}}$, calculated from the measured resonance frequencies are reported in Table 1.

\begin{tabular}{l|cc}
\multicolumn{3}{c}{ Table 1: Natural pulsations of $G_{c o}(s)$ transfer function. } \\
\hline \hline Natural frequency & $\omega_{n_{1}}(\mathrm{~Hz})$ & $\omega_{n_{2}}(\mathrm{~Hz})$ \\
\hline Theoretical simulation values & 2239.9 & 3800.1 \\
Experimental values & 2180.4 & 3517.3 \\
Relative errors & $2.66 \%$ & $7.44 \%$ \\
\hline \hline
\end{tabular}

Static gains $k_{c_{1}}$ and $k_{c_{2}}$ are estimated to give both the correct frequency value for the first antiresonance (which occurs between the two resonances) and the right static gain of the whole system. This identification procedure gives the following transfer polynomials:

$$
\begin{aligned}
N_{c o}(s)= & 2.311 \times 10^{-9} s^{2}+2.22 \times 10^{-6} s+0.08 \\
D(s)= & 8.837 \times 10^{-18} s^{4}+1.553 \times 10^{-14} s^{3}+ \\
& 6.802 \times 10^{-9} s^{2}+4.934 \times 10^{-6} s+1 .
\end{aligned}
$$

The identified response model is compared with experimental responses in frequency (Fig. 7) and time (Fig. 8) domains.

\subsection{Characterization of the electric charge transfer operator}

\subsubsection{Experimental setup for electric charge measurement}

To estimate the electric charges on the sensing electrodes pattern, the measurement electronic device is based on an operational amplifier in a current integrator operating mode (Fig. 9). This electronic setup uses a resistor $R_{c r}$ in parallel to a feedback capacitance $C$. The contribution of this resistance is to discharge the capacitance $C$, to avoid 

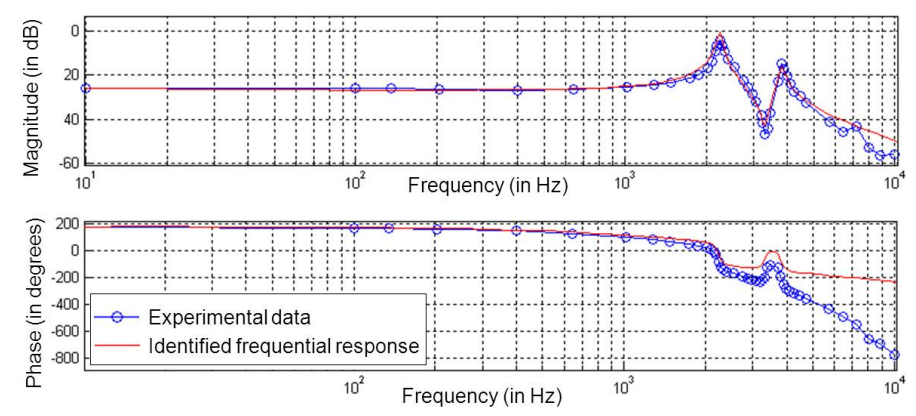

Figure 7: Experimental Bode diagram of the $\frac{\delta}{u}$ transfer function ( $\delta$ in $\mu m$ and $u$ in Volt) and corresponding identified transfer function.

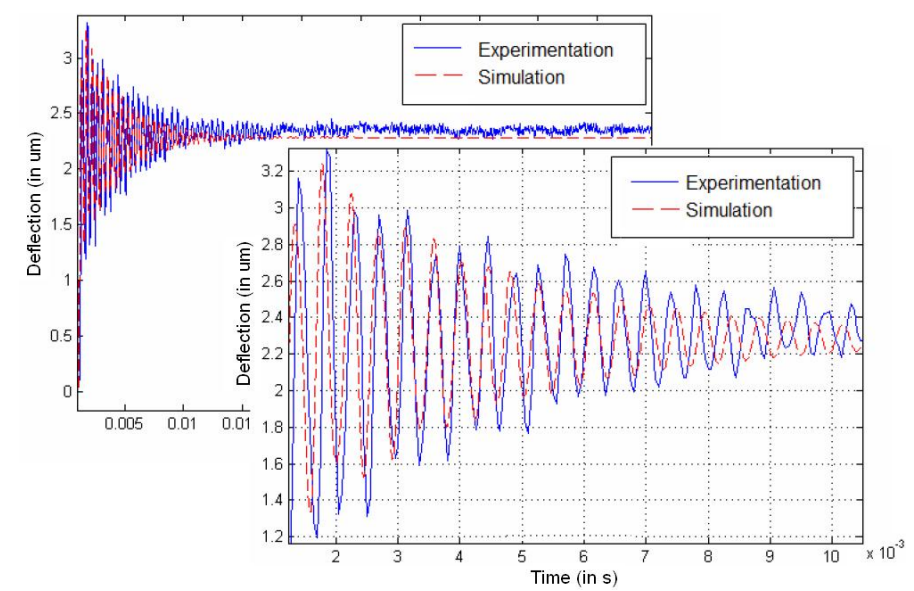

Figure 8: Experimental transient response for the $\frac{\delta}{u}$ transfer operator ( $\delta$ in $\mu m$ and $u$ in Volt) and corresponding identified response.

saturation effects at low frequencies, and to ensure an appropriate working of the electric charges integration. The measured output voltage $V_{s}(s)=\mathcal{L}\left(v_{s}(t)\right)$ is related to the electric charges $Q(s)=\mathcal{L}(q(t))$ in frequency domain as follows:

$$
V_{s}(s)=\mathcal{L}\left(v_{s}(t)\right)=-\frac{1}{C}\left(\frac{s}{s+\frac{1}{R C}}\right) Q(s) .
$$

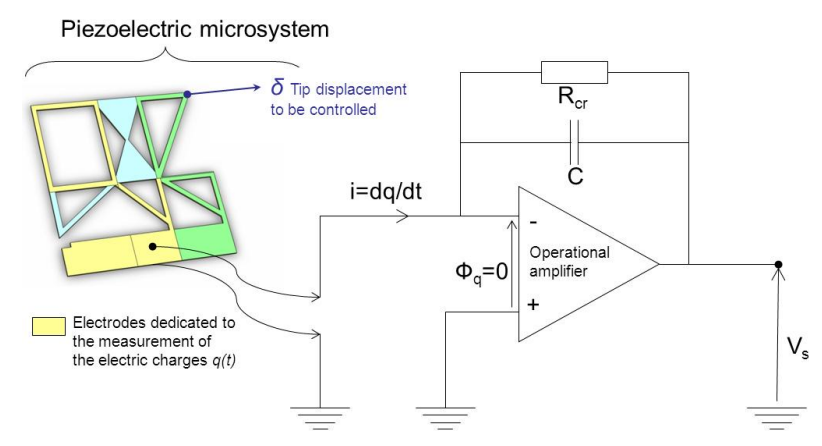

Figure 9: Electronic charge-voltage converter used in the measurement of the electric charges $q(t)$.

The operational amplifier is a micropower precision amplifier (National Semiconductor Corporation $L F 412 A C N$ ), which is characterized by a very low voltage drift with respect to the temperature (max. $\pm 0.4 \mu \mathrm{V} /{ }^{\circ} \mathrm{C}$ ) and a low 
offset voltage (max. $\pm 150 \mu \mathrm{V})$. Its qualities in terms of stability and accuracy as well as its high bandwidth (130 kHz) makes it suitable for measuring nano Coulomb electric charges. The $C$ feedback capacity is chosen equal to $2.2 \mathrm{nF}$, a value close to the experimental capacity of the proprioceptive sensing area of the structure. The value of the resistor $R_{c r}=10 \mathrm{M} \Omega$ must be large enough so as not to disrupt the integration function of the circuit. Along with this request, it cannot be too large so as to avoid the discharge current to pass through it. The choice also depends on the cut-off frequency of the system, which should stay low to avoid the filtering of low frequency components.

\subsubsection{Characterization of the electric charge transfer operator}

The experimental procedure used for the identification of the dynamic behavior of the piezoelectric sensing area is similar to the previous one. A voltage is applied to the actuation area of the structure, while the output of the electronic charge measurement device is recorded. To avoid nonlinear phenomena, the same experimental cautions are taken during this experiment. The modal observability of the first two dominant modes is significant in the charge transfer function $Q(s) / U(s)$ (Fig. 10). The roll-off at higher frequencies is sufficient to consider a simple fourth-order model as a reduced model for $G_{o b}(s)$.

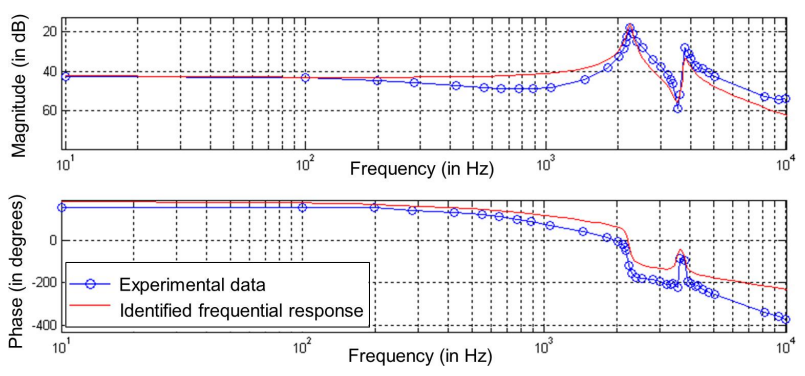

Figure 10: Experimental Bode diagram of the $\frac{q}{u}$ transfer function ( $q$ in $C$ and $u$ in Volt) and corresponding identified transfer function.

\subsubsection{Interests of the $J^{k, t h v}$ criteria for vibrational dynamics identification}

Insights both on frequency and spatial aspects into the pseudo-optimal structure can be given.

- In the frequency domain, if the optimization criterion $J^{k, t h v}$ is satisfied, then the dominant resonance peaks are clearly visible and occur at the same frequency for both deflection transfer function (i.e. modal controllability authority) and charge transfer function (i.e. modal observability authority). This shows that including observability based criterion from the first designing steps results in very effective design where a correspondance between the transfer functions is observed as seen in (Fig. 10). The experimental frequency response functions respects also the specific pattern imposed by our criterion in (Fig. 2). The vibrational dynamics polynomial $D(s)$ for the electric charge transfer function can be chosen equal to that corresponding to the actuation transfer function (11):

$$
\sum_{i=1}^{2} \frac{k_{o_{i}}}{1+\frac{2 \xi_{i}}{\omega_{n i}} s+\frac{1}{\omega_{n i}^{2}} s^{2}}=\frac{N_{o b}(s)}{D(s)}
$$

The computation of the static gains $k_{o_{1}}$ and $k_{o_{2}}$ are based on the identification of the zero (antiresonance) of the experimental charge transfer function and static gain, to give :

$$
N_{o b}(s)=-1.407 \times 10^{-11} s^{2}-1.506 \times 10^{-8} s-0.007
$$

The identified response model of the charge frequency response function matches the experimental frequency (Fig. 11) and time (Fig. 12) responses well.

- Regarding modal spatial shapes associated with $\omega_{n_{1}}$ and $\omega_{n_{2}}$, significant modal strain of the actuation area of the structure implies an important modal displacement of the output (i.e. high peaks amplitudes in the $\frac{\delta}{u}$ frequency response function) on one hand, and an important strain of the sensing piezoelectric beams (high 


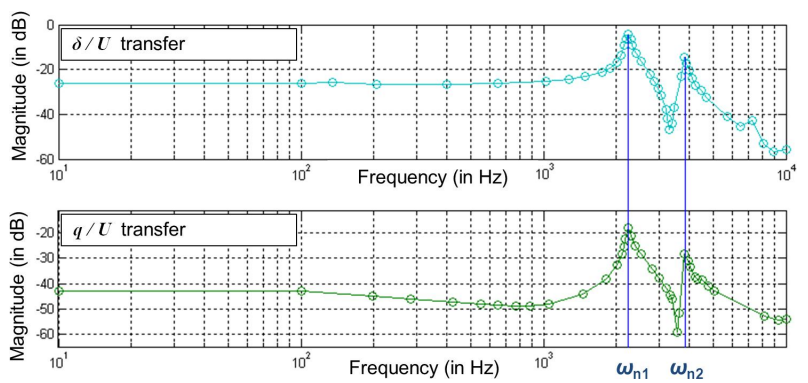

Figure 11: Correspondence of the dominant modes $\omega_{n_{1}}$ and $\omega_{n_{2}}$ in both deflection and charge transfer functions.

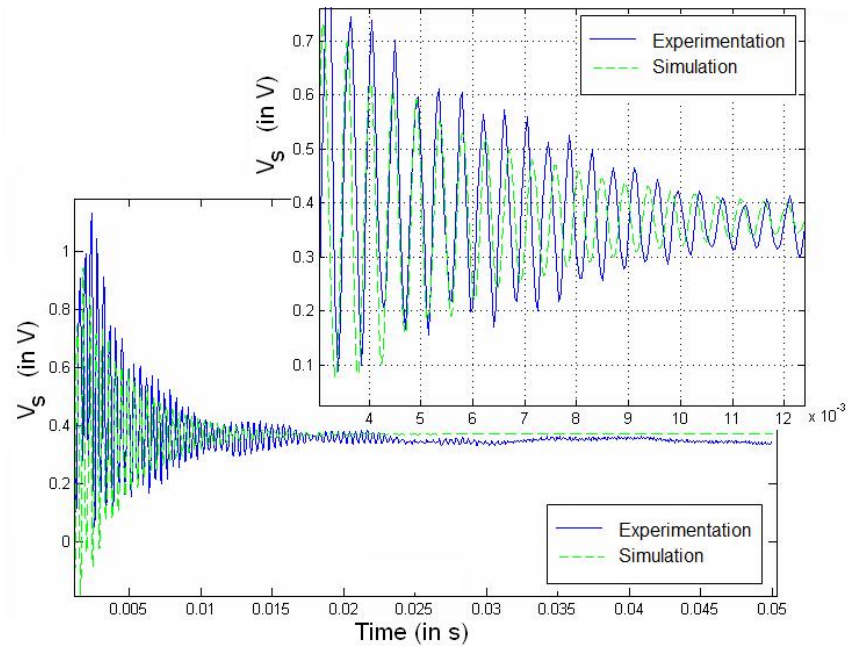

Figure 12: Experimental transient response for the $\frac{V_{s}}{u}$ transfer function ( $V_{s}$ in $V$ and $u$ in Volt) and corresponding identified response along with the electronic instrumentation.

resonance peaks in the electric charge $\frac{q}{u}$ frequency response function) on the other hand (Fig. 13). Thus, such modal configuration explains why dominant resonant modes for deflection are well observed by electric charge measurement within the structure.

\subsection{Linear modal state-space representation}

Good observability of the dominant modes obtained through the criterion $J^{k, t h v}$ leads to vibrational dynamics $D(s)$ that are identical for both charge and deflection transfer functions in the frequency range of interest. These structural properties are of major importance to reconstruct the output displacement. From the identified frequency responses, the vibrational behavior of the whole structure can be reduced to their two dominant flexible modes, each one considered as a second-order transfer function. We recover the modal displacement in the Laplace domain as follows :

$$
z_{i}=\frac{1}{\frac{s^{2}}{\omega_{n_{i}}^{2}}+\frac{2 \xi_{i} s}{\omega_{n_{i}}}+1}, \quad i=1,2
$$

where the index $i$ represents the $i$-th flexible mode. According to (3), the state vector $x=\left(\begin{array}{lllll}\dot{z}_{1} & \omega_{n_{1}} z_{1} & \dot{z}_{2} & \omega_{n_{2}} z_{2}\end{array}\right)^{t}$ is chosen. It leads to the following state space representation of the system for both the deflection and electric charge responses:

$$
\left\{\begin{array}{l}
\dot{\mathbf{x}}=\mathbf{A} \mathbf{x}+\mathbf{B} u \\
y_{c o}=\delta=\mathbf{C}_{\mathbf{c o}} \mathbf{x} \\
y_{o b}=q=\mathbf{C}_{\mathbf{o b}} \mathbf{x}
\end{array}\right.
$$



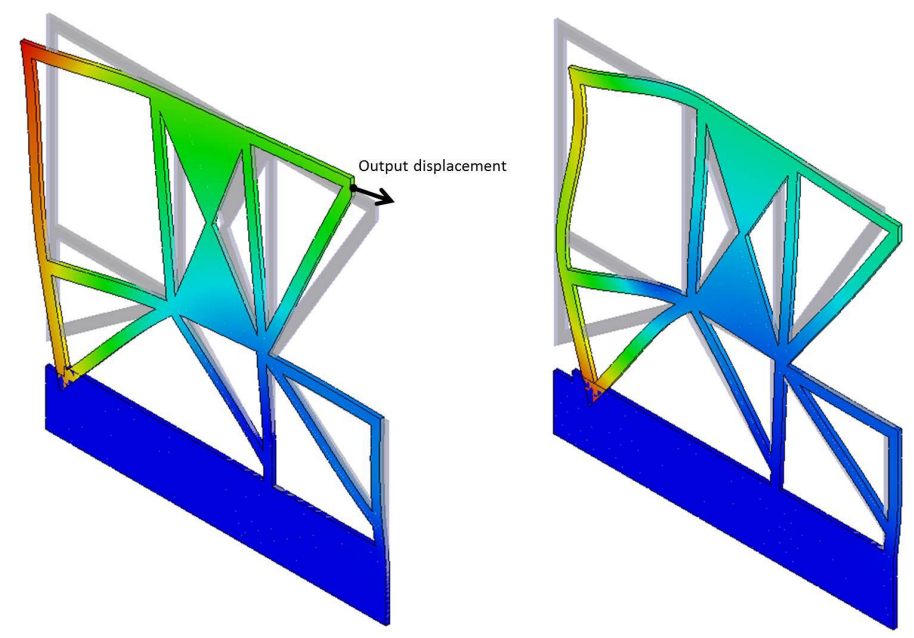

Figure 13: Mode shape plots of the pseudo-optimal structure associated with $\omega_{n_{1}}$ on the left and $\omega_{n_{2}}$ on the right. (Actuation and sensing parts within the structure are reported in Fig. 3.)

with

$$
\begin{aligned}
& \mathbf{A}=\left(\begin{array}{cc|cc}
-2 \xi_{1} \omega_{n_{1}} & -\omega_{n_{1}} & 0 & 0 \\
\omega_{n_{1}} & 0 & 0 & 0 \\
\hline 0 & 0 & -2 \xi_{2} \omega_{n_{2}} & -\omega_{n_{2}} \\
0 & 0 & \omega_{n_{2}} & 0
\end{array}\right) \\
& \mathbf{B}=\left(\begin{array}{ll|ll}
\omega_{n_{1}}^{2} & 0 & \omega_{n_{2}}^{2} & 0
\end{array}\right)^{\mathbf{t}} \\
& \mathbf{C}_{\mathbf{c o}}=\left(\begin{array}{ll|ll}
0 & \frac{k_{c_{1}}}{\omega_{n_{1}}} & 0 & \frac{k_{c_{2}}}{\omega_{n_{2}}}
\end{array}\right) \\
& \mathbf{C}_{\mathbf{o b}}=\left(\begin{array}{ll|ll}
0 & \frac{k_{o_{1}}}{\omega_{n_{1}}} & 0 & \frac{k_{o_{2}}}{\omega_{n_{2}}}
\end{array}\right)
\end{aligned}
$$

\section{Control of the tip deflection}

In previous section, the measurement of the tip deflection was performed using exteroceptive displacement sensor (laser sensor). A reduced electromechanical model of the tip deflection $\delta$ as a function of the input control voltage $u$ was experimentally identified. As these works consider only linear effects of piezoelectricity, the corresponding dynamical models have to be completed to consider nonlinear phenomena in a control-oriented perspective. The hysteresis phenomenon in deflection will be identified and then compensated in this section. A cascaded High Authority Control (HAC) / Low Authority Control (LAC) controller with damping feature will also be proposed and synthesized. Finally, this full control scheme will be implemented to ensure the performances required in micromanipulation tasks.

In the following subsection, we take advantage of the proposed observability-based criteria $J^{k, t h v}$ to complete the control scheme with an observer. The relationship between the total electric charge $q$ and the control voltage $u$ is investigated. Finally, the estimated tip deflection $\hat{\delta}$ can be reconstructed efficiently through a modal state observer. This one exploits the proprioceptive sensing property of our pseudo-optimally-design device.

\subsection{Control using exteroceptive laser sensor measurement}

\subsubsection{Nonlinear phenomena analysis}

The deflection is non-linearly linked to applied voltage $u$ through the hysteresis effect and the creep phenomena in a decoupled way. Hysteretical behavior depends on the past and present values of $u$ but also on its frequency [21].

1. The creep phenomenon is the drift of the deflection observed after the transient part, when a step voltage is applied to the piezoelectric actuator. It can be considered as an additional behavior occurring when the steadystate is reached, so that it is often modeled as a simple delayed transfer operator [21]. Experimental results 
show that the transient part of the piezoelectric microactuator is shorter than $300 \mathrm{~ms}$ whereas the creep settling time is longer than $180 \mathrm{~s}$. As a consequence, the creep will be considered as a disturbance that must be rejected by the controller [22].

2. A $\pm 80 \mathrm{~V}$ sine voltage input is applied to the system, and the free displacement output $\delta$ at the tip of the device is recorded.Experimental results of the Fig. 14 show the phase shift and the shape variation due to the mechanical linear vibrational dynamics $G_{c o}(s)$ [23]. The experimental static linear deflection is closed to the value predicted by this method (static gain $\left.k_{s}=N_{c o}(s=0)=0.08 \mu \mathrm{m} / \mathrm{V}\right)$.

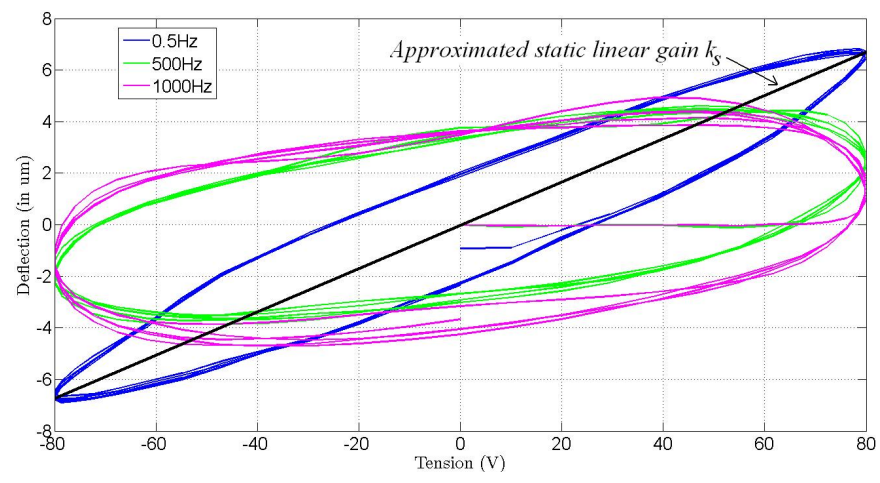

Figure 14: Measured deflection hysteresis of the piezoelectric actuator for various frequency voltage inputs.

According to [24], we propose to model hysteresis phenomena with a static hysteresis operator $H_{s}(u)$ of a constant shape in series with a linear dynamical operator with an unitary static gain (Fig. 15). Indeed, it was already shown by authors in [25] that the linear vibrational characteristics of the transient response is independent of the amplitude of the voltage. Thus, in the following, the transient part will be assumed to be the normalized $G_{c o}$ transfer function (i.e. with an unitary static gain).

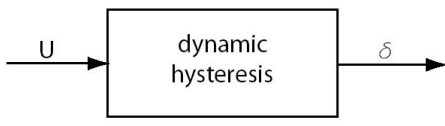

(a)

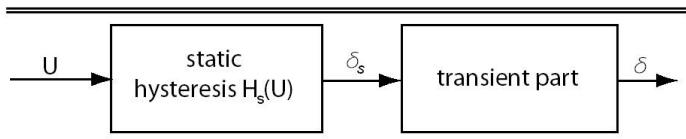

(b)

Figure 15: Dynamic hysteresis equivalence.

\subsubsection{Hysteresis compensation using the Prandtl-Ishlinskii (PI) static hysteresis model}

To perform the linearization, the inverse static model $H_{s}^{-1}(u)$ is put in cascade with the system (Fig. 16). The Prandtl-Ishlinskii (PI) model has been chosen due to its simplicity and accuracy [26]. This one is based on the superposition of several elementary backlash operators characterized by their threshold $r$ and weighting coefficient $h$. Let $H_{r_{i}}\left[u, \delta_{i 0}(t)\right]$ be the $i$-th backlash operator characterized by its threshold $r_{i}$ and initial state $\delta_{i 0}$. The static deflection $\delta_{s}$ is given by the following equation according to PI operator:

$$
\delta_{s}=\Gamma(u)=[h]^{t} \cdot\left[H_{r_{H}}\left[u, \delta_{H_{0}}\right](t)\right],
$$

where $H_{r_{H}}\left[u, \delta_{H_{0}}\right](t)=\left[H_{r_{0}}\left[u, \delta_{0_{0}}\right](t) \ldots H_{r_{n}}\left[u, \delta_{n_{0}}\right](t)\right]^{t}$ is the $(n+1)$ vector of backlash operators and $[h]=\left[h_{0} \ldots h_{n}\right]^{t}$ is the weighting factor. 


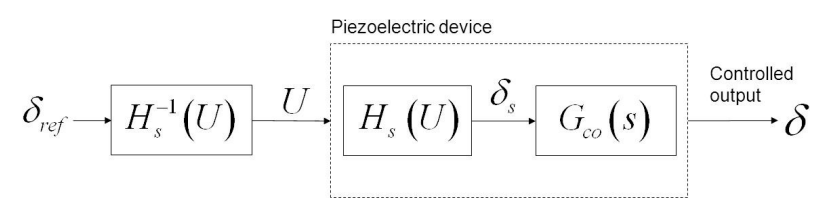

Figure 16: Scheme of the hysteresis compensation.

The computation of the inverse PI model is another static PI hysteresis, considering that the deflection is the input and the voltage is the output. Based on the same principle as the direct PI model, the new thresholds $r_{i}^{\prime}$ and weighting elements $h_{i}^{\prime}$ are given as follows :

$$
r_{i}^{\prime}=\sum_{j=0}^{i} h_{j}\left(r_{i}-r_{j}\right), \quad i=0, \ldots, n
$$

and

$$
\begin{aligned}
& h_{0}^{\prime}=\frac{-1}{h_{0}} \\
& h_{i}^{\prime}=\frac{-h_{i}}{\left(h_{0}+\sum_{j=1}^{i} h_{j}\right)\left(h_{0}+\sum_{j=1}^{i-1} h_{j}\right)}, \quad i=1, \ldots, n .
\end{aligned}
$$

The PI model has been identified with 18 backlash operators, considering a $80 \mathrm{~V}$ sine voltage applied to the piezoelectric device. The output tip displacement $\delta$ is directly measured from the laser sensor. A $0.5 \mathrm{~Hz}$ low frequency, far below the resonant frequencies, has been selected for experiments to avoid the dynamic effects on the hysteresis shape. Following the identification procedure (Fig. 17), the inverse model is implemented in the controller and shows a good effectiveness in the compensation of the hysteresis (Fig. 18).

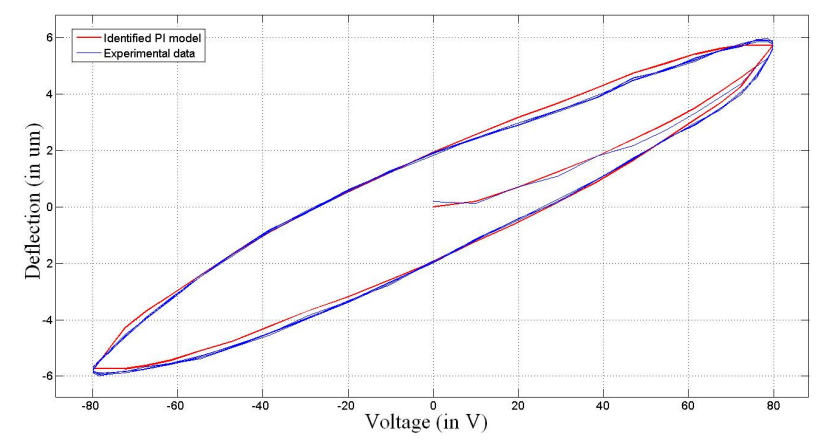

Figure 17: Identification of the static experimental hysteresis.

\subsubsection{A HAC/LAC damping strategy for in-plane motion control}

This approach combines two different loops as shown on Fig. 19: the active damping regulator $H_{l}$ (LAC) reduces the settling time of transient response, while a (HAC) compensator $H_{L}$ achieves integral action at low frequency and significant modifications to the open-loop system poles. The HAC controller deals with the trade-off between the conflicting requirements of performances-robustness-stability. The outer loop of the compensator is designed on the damped structure.

If we consider that the two first vibrational modes are dominant, i.e. reduced identified model can efficiently approximate the full model, a very simple LAC controller can be designed. This one provides good intrinsic stability properties for such king of systems. Considering the trajectory of the closed-loop system poles in the complex plane, the use of a pure gain $H_{l}(s)=g=7$ as a feedback $H_{l}$ controller is chosen to decrease the influence of the first resonant 

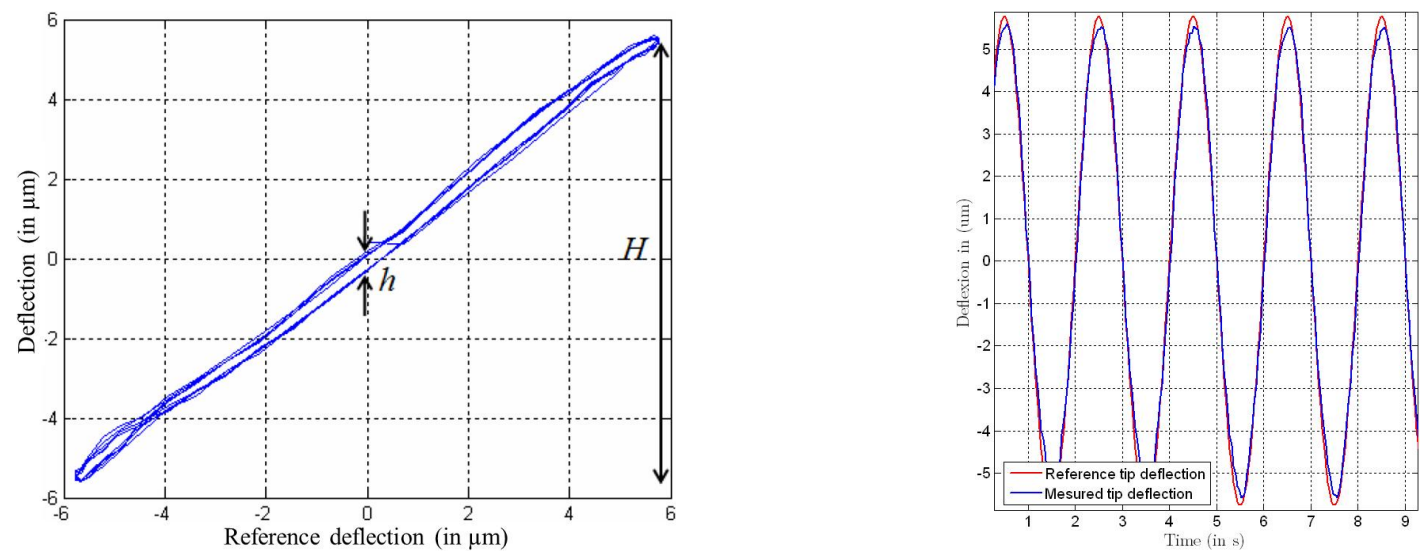

Figure 18: Experimental results of hysteresis linearization in quasi-static (before PI compensation - maximum hysteresis of $31 \%$ and after PI compensation - maximum hysteresis of 3\%) and low-frequency sine input trajectory reference tracking $\delta_{r}(t)$.

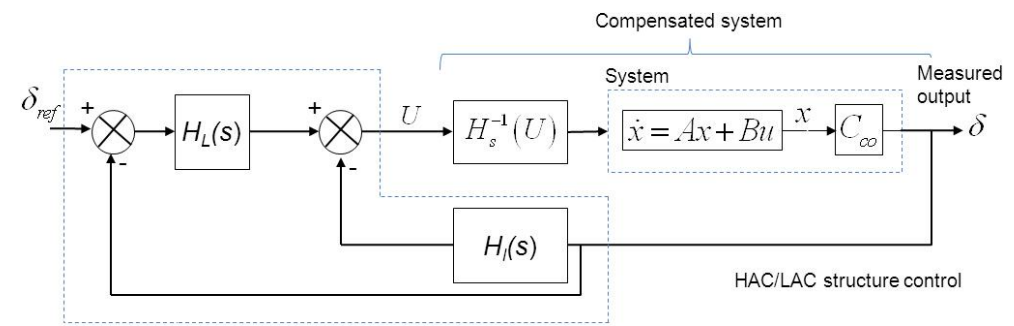

Figure 19: Scheme control of the cascaded HAC/LAC loops.

mode of the compensated system (Fig. 20). This gain value is chosen based on simulation analysis through the root locus of the system.

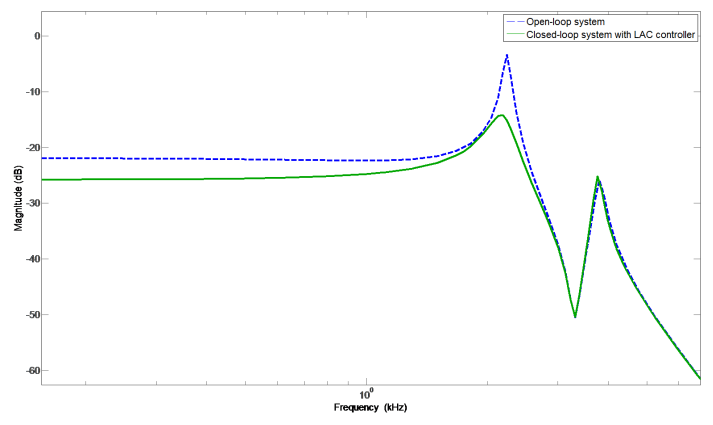

Figure 20: Bode diagrams in magnitude of the open-loop transfer $\delta(s) / u(s)$ and pre-compensated closed-loop transfer with $H_{l}(s)$.

To increase the gain at low frequency, the HAC compensator includes a simple integral action, to eliminate the static error and to reject disturbance. At high-frequency, it provides sensor noise rejection and stability robustness. A simple multiplicative term has been added to the integrator $H_{L}(s)=k_{H_{L}} / s$ to increase the bandwidth of the precompensated loop transfer up to $220 \mathrm{rad} / \mathrm{s}$.

$$
L_{c o}(s)=\frac{H_{L}(s) G_{c o}(s)}{1+H_{l}(s) G_{c o}(s)}
$$

The resulting regulator provides interesting gain and phase margins, $M_{g}=23 \mathrm{~dB}$ and $M_{\varphi}=82 \mathrm{deg}$ at $\omega_{P}=256 \mathrm{rad} / \mathrm{s}$ 
respectively. Steps were experimentally applied to the device to validate the performances of the whole HAC/LAC controller along with the P.I. compensator, providing a settling time of $t_{5 \%}=12.5 \mathrm{~ms}$ (Fig. 21 ). Let us note that the bandwidth that is reached by our low-order control scheme is compatible with micromanipulation tasks requirements and similar to bandwidth of other control schemes [27] [21].

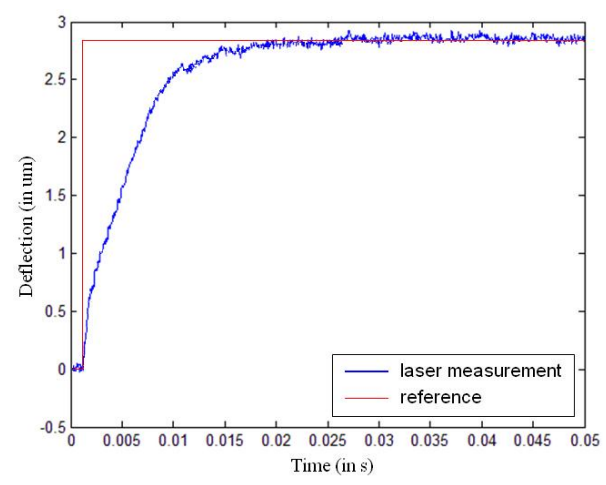

Figure 21: Experimental step response to a 2.8 $\mu \mathrm{m}$-amplitude reference for the HAC/LAC compensated system.

\subsection{Control using proprioceptive electric charge observation}

In this section, we present the design of a position observer that take into account the benefits obtained by the observability-based criteria $J^{k, t h v}$ during the design of the system. This observer permits to efficiently reconstruct the tip deflection estimate $\hat{\delta}$ through a modal state observer based on the proprioceptive sensor (Fig. 5). The algorithm uses a modal observer to reconstruct the linear dynamics of the tip deflection. This one is completed with additional features to take into account nonlinearities that may occur in the piezoelectric induction of electric charges.

\subsubsection{Observer poles placement}

To observe the linear dynamics of $G_{o b}(s)$, a Luenberger state observer, is implemented as:

$$
\left\{\begin{array}{l}
\dot{\hat{\mathbf{x}}}=\mathbf{A} \hat{\mathbf{x}}+\mathbf{B} u+\mathbf{K}_{\mathbf{o b}}\left(y_{o b}-\hat{y}_{o b}\right) \\
\hat{y}_{o b}=\hat{q}=\mathbf{C}_{\mathbf{o b}} \hat{\mathbf{x}}
\end{array}\right.
$$

The observer gains of the $(4 \times 1)$ vector $\mathbf{K}_{\mathbf{o b}}$ are chosen such that the prediction error $\mathbf{e}=\mathbf{x}-\hat{\mathbf{x}}$ vanishes according to $\dot{\mathbf{e}}=\left(\mathbf{A}-\mathbf{K}_{\mathbf{o b}} \mathbf{C}_{\mathbf{o b}}\right) \mathbf{e}$.

Robustness analysis of the whole observer-based control scheme is performed. The HAC/LAC control action is expressed in the Laplace domain as follows:

$$
u(s)=H_{L}(s)\left(\delta_{r}-\delta\right)-H_{l}(s) \delta
$$

According to previous section, $H_{l}(s)=g$ and $H_{L}(s)=k_{H_{L}} / s$ and thus:

$$
u(s)=\frac{k_{H_{L}}}{s} \delta_{r}-\left(g+\frac{k_{H_{L}}}{s}\right) \mathbf{C}_{\mathbf{c o}} \hat{\mathbf{x}}
$$

The open-loop system including modal state observer and controller is then deduced from (24) and (26) as:

$$
L_{o b}(s)=G_{o b}(s) K(s)
$$

where

$$
u(s)=K(s) y_{o b}(s)=-\left(g+\frac{k_{H_{L}}}{s}\right) \mathbf{C}_{\mathbf{c o}} \mathbf{K}_{\mathbf{o b}}\left(s \mathbb{I}-\mathbf{A}+\mathbf{K}_{\mathbf{o b}} \mathbf{C}_{\mathbf{o b}}+\left(g+\frac{k_{H_{L}}}{s}\right) \mathbf{B} \mathbf{C}_{\mathbf{c o}}\right)^{-1} y_{o b}(s)
$$

The four gains of the $\mathbf{K}_{\mathbf{o b}}$ vector are computed following a root-clustering procedure, with an observation dynamics four times faster than the control dynamics. In addition, observation poles are chosen real to avoid oscillations. The resulting regulator provides interesting gain and phase margins, $M_{g}=26 \mathrm{~dB}$ and $M_{\varphi}=77 \mathrm{deg}$ respectively. 


\subsubsection{Non-linearities modeling and compensation}

Electric charges modeling is a linear function of the mechanical strain in piezoelectric materials [28]. Nevertheless and as it was previously shown, there is an hysteretical behavior between applied voltage and the actuated beams strain. But this non-linearity affects also sensing parts strain, because actuation deforms the whole structure, and therefore affects the strain of the sensing parts. The hysteresis non-linearity between applied voltages and controlled output $y_{c o}=\delta$ was efficiently corrected with the help of PI operator $H_{s}^{-1}$. Nevertheless, as measured output $y_{o b}=q$ are not controlled output $y_{c o}=\delta$, this compensation is not able to linearize the transfer between the observed output $y_{o b}$ and the input applied voltage $u$ (Fig. 22).
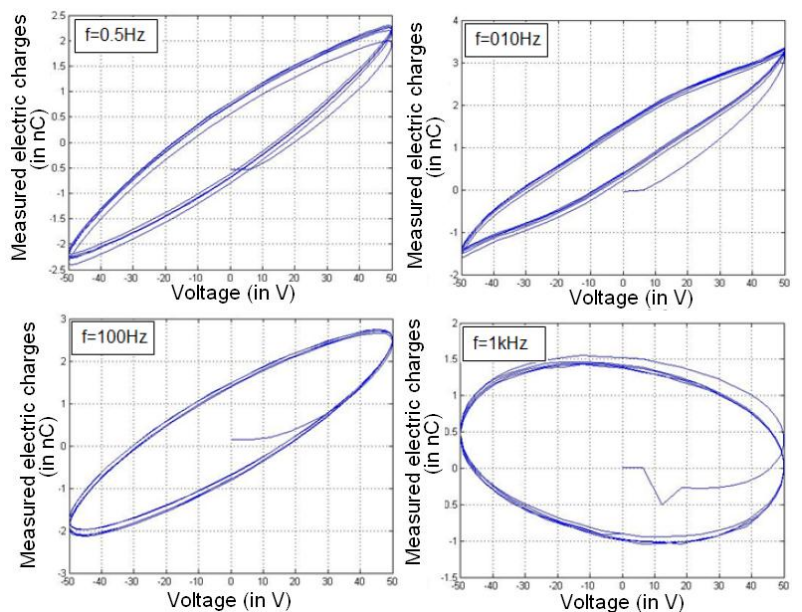

Figure 22: Measured charge hysteresis of the sensitive area of the system for various frequency voltage inputs.

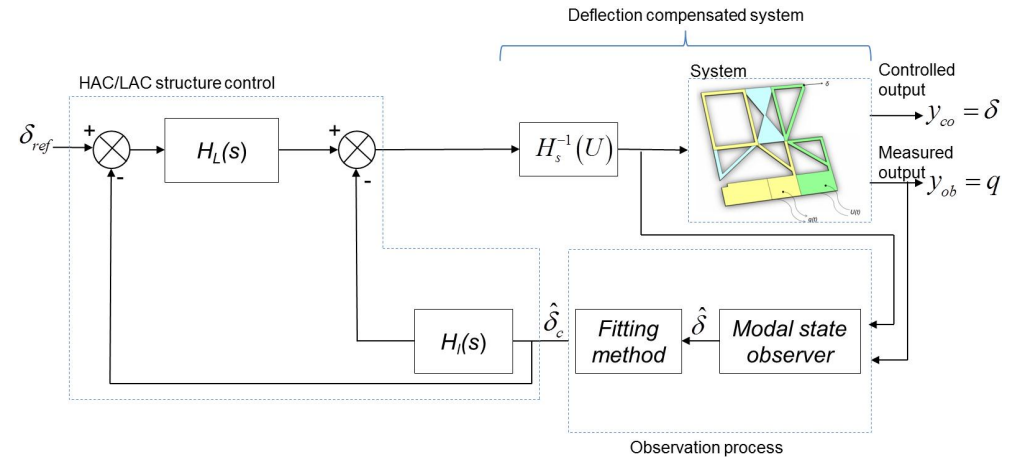

Figure 23: Complete observation/control scheme based on proprioceptive measurement.

The non-linearity compensation of $q / u$ transfer operator have to be done in a second step after the reconstruction of the output $y_{c o}=\delta$ as $\hat{\delta}$. A curve fitting technique is used to linearize the steady-state hysteresis phenomenon that affect the charge induction. This compensation algorithm permits a fast and efficient corrective action (Fig. 23). The modal state observer estimates the linear contribution of the $\hat{\delta}$ deflection through the $K_{o b}$ matrix. It captures the transient part of the deflection. The observer output $\hat{\delta}$ is then corrected by the curve fitting technique into a new deflection $\hat{\delta}_{c}$ which is send to the controller. A characterization process that maps $\hat{\delta}$ into $\hat{\delta}_{c}$ was achieved off-line, considering all the various signals combination of variable amplitudes, with a $0.2 \mu \mathrm{m}$ resolution compatible with the manipulation requirements. The values of the steady-state deflection $\hat{\delta}_{c}$ (measured with exteroceptive laser sensor) and the estimated deflection $\hat{\delta}$ (estimated with the Luenberger observer) are collected and compared for each set point stage, and classified in rising and falling edges. Each ratio $r_{i j}=\frac{\delta_{c_{i j}}}{\hat{\delta}_{i j}}$ is calculated and stored into a table for interpolation. It depends on the index $i$ of the considered stage set point, and the index $j$ on whether the current reference is rising or falling (equal to 1 or 
2). Then, in operating mode, the corrected $\hat{\delta}_{c}$ deflection is computed on-line according to $\hat{\delta}_{c_{i j}}=r_{i j} \hat{\delta}_{i j}$. Experimental results of the full observation process, i.e. linear state observer and compensation, shows the effectiveness of the proposed method to reconstruct the tip deflection: the vibrational dynamic is well captured and the static nonlinear effect is well compensated (as can be shown on Fig. 24).

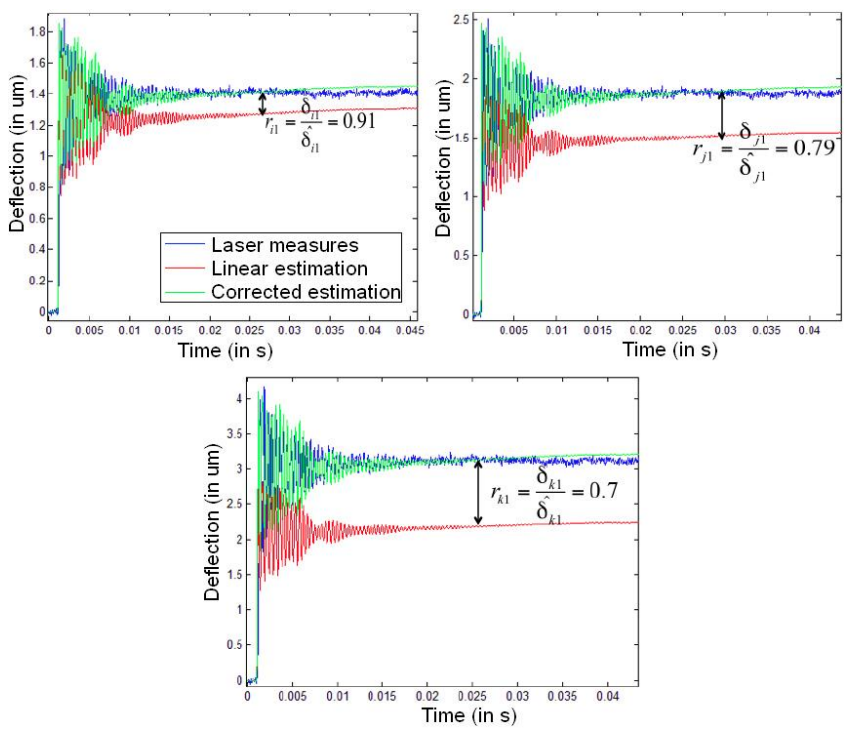

Figure 24: Step responses of the deflection for different operating voltages.

\subsubsection{Experimental results of the deflection control using proprioceptive observation}

The whole control system depicted on the Fig. 23 was tested with a step position reference signal. The response of the experimental system does not exhibit any overshoots, and the response time is approximately $t_{5 \%}=13 \mathrm{~ms}$. Furthermore, the use of an observer tends to filter the measurement noise phenomenon which appears when the exteroceptive laser sensor is used (Fig. 25). Using proprioceptive information seems therefore very attractive for the end-effector control. To emphasize the effectiveness of this observer/controller architecture, reference steps of various amplitudes were experimentally applied to the device. For each of theses reference signals, the tracking performances are validated without overshoot and the deflection hysteresis is also well compensated (Fig. 25).
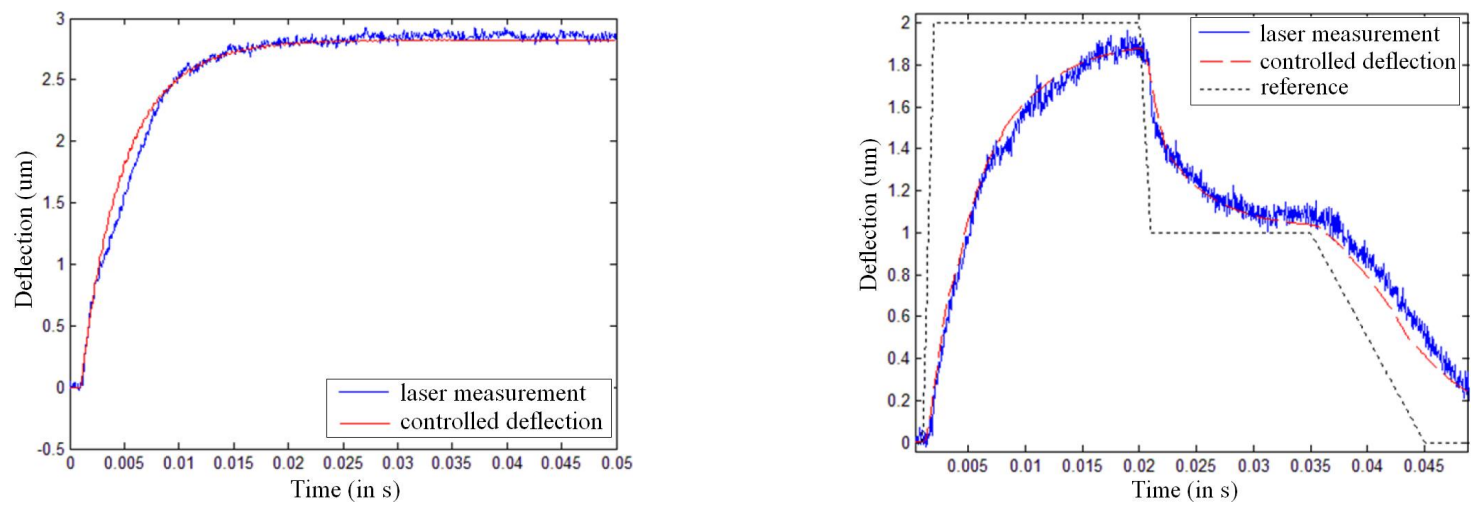

Figure 25: Experimental step response (on the left) and tracking response (on the right) for the HAC/LAC system including observer and non-linear compensation (Fig. 23) 


\section{Discussions and conclusions}

In the micromechatronic field, designers of integrated systems are faced with complex multi-objective design problems. The strategy proposed in this paper led to the design of a compliant microactuator that optimally integrates actuating and sensing areas in monolithic structures. We took advantage of versatile criteria to synthesize high performance microrobotic flexible mechanism designs. In addition to classical mechanical criteria currently encountered in topology optimization (i.e. force and displacement maximization), it is of great interest to consider efficient observability-based criteria simultaneously. These criteria can fully and optimally integrate the actuation and sensor capabilities into the structure, as regards to the performances achievable by the controller.

Considering dynamic effects from the first design steps is of a particular interest when dealing with flexible structures as it was demonstrated for the design of a piezoelectric microgripper. Indeed, taking advantage of our frequencybased criterion, the dominant vibrational modes which govern the tip deflection of the device are now fully observable from the integrated sensing area of the device. From a topological point of view, considering observability-based criteria to shape the open-loop frequency response of both the controlled deflection and observed electric charges gives an efficient and well designed full microrobotic device. In particular, this effectiveness allows an efficient and confident observer-based reconstruction of the tip-deflection.

Regarding the reduced modal content of the deflection transfer function, a very simple HAC/LAC control approach can ensure good performance in tracking. Even if HAC/LAC methods are not new in control literature, this paper is one of the few to show its power for flexible microactuators control. In comparison with classic robust control approaches, this one is competitive in terms of robustness and performances. Based on the pseudo-optimal proprioceptive sensing capabilities of our device, a simple modal observation process reconstructs efficiently the dynamics of the deflection of the microactuator.

More complex charge integration techniques for low frequency signals could be considered in future works. In particular, electronic circuitry based on analog-to-digital converter could be used to integrate and to numerically capture peak values of electric charge signal that is generated by the sensor when subjected to a static load. Measurement accuracy could also be improved by utilizing feedback techniques to compensate for the signal loss due to charge leakage.

The problem of high-pass filter created by capacitive source impedance of piezoelectric sensors can also be overcome by using a low-frequency bypass technique [29]. In this approach, the low-frequency component of the measured signal is replaced by an estimate based on the system input and an open-loop model. The actual output of the piezoelectric sensor is complemented with an estimate at frequencies below the crossover frequency and do not add dynamics to the control loop.

The optimal integration of piezoelectric actuation and sensor capabilities within the structure from both the mechanical and control-/observation- points of view illustrates the concept of smart adaptronic system. Natural perspective of these works are the possible integration of this control structure into system-on-chip.

\section{References}

[1] J. M. Breguet, al., Monolithic piezoceramic flexible structure for micromanipulation, in: 9th International Precision Engineering Seminar and 4th International Conference on Ultraprecision in Manufacturing Engineering (IPES-UME'1997), Braunschweig, Germany, 1997, pp. 397-400.

[2] D. Campolo, R. Sahai, R. S. Fearing, Development of piezoelectric bending actuators with embedded piezoelectric sensors for micromechanical flapping mechanisms, in: IEEE Int. Conf. on Robotics and Automation (ICRA 2003), Taipei, Taiwan, 2003 , pp. 3339-3346.

[3] P. J. C. Branco, J. A. Dente, On the electromechanics of a piezoelectric transducer using a bimorph cantilever undergoing asymmetric sensing and actuation, Smart Materials and Structures 13 (2004) 631-642.

[4] M. Grossard, N. Chaillet, S. Regnier, Flexible Robotics: Applications to Multiscale Manipulations, Wiley, 2013.

[5] H. Janocha, Adaptronics and Smart Structures - Basics, Materials, Design and Applications, 2nd ed., Springer-Verlag, 2007.

[6] J. H. Han, I. Lee, Optimal placement of piezoelectric sensors and actuators for vibration control of composite plate using genetic algorithms, Smart Materials and Structures 8 (1999) 257-267.

[7] M. Moallem, M. R. Kermani, R. V. Patel, M. Ostojic, Control of a positioning system with structural flexibility using piezoelectric transducers, in: 42nd IEEE Conference on Decision and Control (CDC'2003), Vol. 5, 2003, pp. 4505-4510.

[8] S. Leleu, H. Abou-Kandil, Y. Bonnassieux, Piezoelectric actuators and sensors location for active control of flexible structures, IEEE Trans. on Instrumentation and Measurement 50(6) (2001) 1577-1582.

[9] E. Silva, S. Nishiwaki, N. Kikuchi, Design of piezocomposite materials and piezoelectric transducers using topology optimization - part ii, Computational Methods in Engineering 6 (1999) 191-222. 
[10] S. Wang, K. Tai, S. T. Quek, Topology optimization of piezoelectric sensors/actuators for torsional vibration control of composite plates, Smart Materials and Structures 15 (2006) 253-269.

[11] M. Abdalla, al., Design of a piezoelectric actuator and compliant mechanism combination for maximum energy efficiency, Smart Material and Structures 14 (2005) 1421-1430.

[12] M. Maddisetty, M. Frecker, Dynamic topology optimization of compliant mechanisms and piezoceramic actuators, ASME Journal of Mechanical Design 126 (2002) 975-983.

[13] B. Zheng, C.-J. Chang, H. G. Gea, Design of piezoelectric actuator with in-plane motion using topology optimization, in: ASME International Design Engineering Technical Conferences and Computers and Information in Engineering Conference (IDETC/CIE'2007), Las Vegas, Nevada, USA, 2007.

[14] B. Zheng, C. Lu, H. Z. Huang, Topology optimization of piezoelectric force sensor, in: International Conference on Apperceiving Computing and Intelligence Analysis (ICACIA'2008), 2008, pp. 132-136.

[15] H. Du, al., Topological optimization of mechanical amplifiers for piezoelectric actuators under dynamic motion, Smart Material and Structures 9 (2000) 788-800.

[16] R. El Khoury Moussa, M. Grossard, N. Chaillet, M. Boukallel, A. Hubert, Optimal design and control simulation of a monolithic piezoelectric microactuator with integrated sensor, in: IEEE/ASME International Conference on Advanced Intelligent Mechatronics (AIM'2010), 2010, pp. $420-425$.

[17] E. Jonckheere, Principal components analysis of flexible systems - open-loop case, IEEE Trans. on Automatic Control 29 (1984) $1095-1097$.

[18] R. El Khoury Moussa, M. Grossard, M. Boukallel, A. Hubert, N. Chaillet, Optimal observability-based modelling, design and characterization of piezoelectric microactuators, Smart Materials and Structures 22 (7) (2013) 075036.

[19] B. J. G. Vautier, S. O. R. Moheimani, Charge-driven piezoelectric actuators for structural vibration control: Issues and implementation, Smart Materials and Structures 14 (4) (2005) 575-586.

[20] W. Gawronski, K. B. Lim, Balanced control of Flexible structures, Springer-Verlag, 1996.

[21] M. Rakotondrabe, Y. Haddab, P. Lutz, Quadrilateral modelling and robust control of a non-linear piezoelectric cantilever, IEEE Transactions on Control Systems Technology 17(3) (2009) 528-539.

[22] M. Rakotondrabe, Y. Haddab, P. Lutz, Non-linear modeling and estimation of force in a piezoelectric cantilever, in: IEEE/ASME Internationale Conference on Advanced Intelligent Mechatronics (AIM'2007), 2007.

[23] S. Devasia, E. Eleftheriou, S. O. Moheimani, A survey of control issues in nanopositioning, IEEE Transactions on Control Systems Technology 15 (2007) 802-823.

[24] D. Croft, G. Shed, S. Desavia, Creep, hysteresis and vibration compensation for piezoactuators: atomic force microscopy applications, Journal of Dynamic Systems, Measurements and Control 123(1) (2001) 35-43.

[25] M. Grossard, M. Boukallel, N. Chaillet, C. Rotinat-Libersa, Modeling and robust control strategy for a control-optimized piezoelectric microgripper, IEEE/ASME Transactions on Mechatronics 16 (2010) 674-683.

[26] M. Rakotondrabe, C. Clevy, P. Lutz, Complete open loop control of hysteretic, creeped, and oscillating piezoelectric cantilevers, IEEE Transactions on Automation Science and Engineering 7(3) (2009) 440-450.

[27] S. Khadraoui, M. Rakotondrabe, P. Lutz, Interval modeling and robust control of piezoelectric microactuators, Control Systems Technology, IEEE Transactions on 20 (2) (2012) 486-494.

[28] A. Preumont, Vibration control of active structures: an introduction, 2nde Ed., Kluwer academic publishers, 2002.

[29] Y. K. Yong, A. Fleming, S. Moheimani, A novel piezoelectric strain sensor for simultaneous damping and tracking control of a high-speed nanopositioner, Mechatronics, IEEE/ASME Transactions on 18 (3) (2013) 1113-1121. 